\title{
Rapamycin-mediated mTOR inhibition uncouples HIV-1 latency reversal from cytokine-associated toxicity
}

\author{
Alyssa R. Martin, ${ }^{1}$ Ross A. Pollack, ${ }^{2}$ Adam Capoferri, ${ }^{2,3}$ Richard F. Ambinder, ${ }^{1}$ Christine M. Durand, ${ }^{2}$ and Robert F. Siliciano ${ }^{2,3}$ \\ 1Department of Pharmacology and Molecular Sciences, and ${ }^{2}$ Department of Medicine, Johns Hopkins University School of Medicine, Baltimore, Maryland, USA. ${ }^{3}$ Howard Hughes Medical Institute, \\ Baltimore, Maryland, USA.
}

\begin{abstract}
Current strategies for HIV-1 eradication require the reactivation of latent HIV- 1 in resting CD4+ T cells (rCD4s). Global T cell activation is a well-characterized means of inducing HIV-1 transcription, but is considered too toxic for clinical applications. Here, we have explored a strategy that involves a combination of immune activation and the immunosuppressive mTOR inhibitor rapamycin. In purified rCD4s from HIV-1-infected individuals on antiretroviral therapy, rapamycin treatment downregulated markers of toxicity, including proinflammatory cytokine release and cellular proliferation that were induced after potent $T$ cell activation using $\alpha C D 3 / \alpha C D 28$ antibodies. Using an ex vivo assay for HIV-1 mRNA, we demonstrated that despite this immunomodulatory effect, rapamycin did not affect HIV-1 gene expression induced by T cell activation in these rCD4s. In contrast, treating activated rCD4s with the immunosuppressant cyclosporin, a calcineurin inhibitor, robustly inhibited HIV-1 reactivation. Importantly, rapamycin treatment did not impair cytotoxic T lymphocyte (CTL) recognition and killing of infected cells. These findings raise the possibility of using rapamycin in conjunction with $\mathrm{T}$ cell-activating agents in HIV-1 cure strategies.
\end{abstract}

\section{Introduction}

HIV-1 persists in a latent reservoir in resting memory $\mathrm{CD} 4^{+} \mathrm{T}$ cells despite combination antiretroviral therapy (cART) (1-3). Latently infected cells represent a major barrier to eradication. Integrated proviruses in resting $\mathrm{CD}^{+} \mathrm{T}$ cells $(\mathrm{rCD} 4 \mathrm{~s})$ are minimally transcribed, and thus this reservoir evades immune surveillance. A paradigm for HIV-1 cure (4) involves reactivation of proviral expression in latently infected cells to allow their elimination by immune mechanisms including $\mathrm{CD}^{+}$cytotoxic T lymphocytes (CTLs) (5). Agents eliciting global T cell activation were used in the original detection of the reservoir $(1,2)$ and effectively reverse latency. However, in clinical settings they induce severe adverse reactions by causing systemic release of proinflammatory cytokines $(6,7)$. Although clinical trials using T cell-activating agents such as anti-CD3 and IL-2 have demonstrated transient increases in viremia and changes in HIV-1 intracellular RNA levels (6), this approach has been abandoned in favor of latency-reversing agents (LRAs) that induce HIV-1 transcription without cellular activation. No single LRA reactivates HIV-1 gene expression to levels induced by T cell activation (8). The most effective single agents are PKC agonists, which affect a subset of the pathways stimulated by T cell activation (9). Thus, effective latency reversal may require some immune activation. Here, we explore the idea that immunomodulatory compounds in conjunction with $\mathrm{T}$ cell activation may limit toxicity while allowing maximal reactivation of latent HIV-1.

Conflict of interest: The authors have declared that no conflict of interest exists Submitted: July 13, 2016; Accepted: November 30, 2016.

Reference information: J Clin Invest. 2017;127(2):651-656.

https://doi.org/10.1172/JCl89552.
Adverse reactions elicited by the activation of large numbers of T cells are typically attributed to "cytokine storm" (6). A classic example is toxic shock syndrome, caused by a staphylococcal superantigen that activates all cells expressing a V $\beta 2$ T cell receptor (TCR) (about 10\% of total T cells) (10). Additionally, agents that induce measurable in vitro lymphocyte proliferation have toxicity in vivo. This was dramatically demonstrated by the CD28 superagonist TGN1412, for which other in vitro measures of immune toxicity failed to predict the widespread nonspecific immune activation observed in vivo (11).

We asked whether immunosuppressive agents could block the cytokine production and $\mathrm{T}$ cell proliferation induced by $\mathrm{T}$ cell activation without blocking HIV-1 latency reversal or subsequent killing of infected cells. Many immunosuppressive compounds affect upstream regulators of $\mathrm{T}$ cell activation pathways, and thus affect HIV-1 transcription because of similarities between the HIV-1 long-terminal repeat (LTR) and the promoters of key genes upregulated by T cell activation, including IL2 (12). The widely used immunosuppressant cyclosporin inhibits the calcium-dependent phosphatase calcineurin, blocking nuclear translocation of the transcription factor NFAT, which acts on the IL2 promoter and the HIV-1 LTR (13). Cyclosporin inhibits HIV-1 expression in a cell line model of latency (14).

To avoid inhibition of transcription factors that act on the HIV-1 promoter, we explored immunosuppressants that do not affect signaling immediately downstream of TCR engagement. Rapamycin (sirolimus), an inhibitor of the mammalian target of rapamycin (mTOR), mediates immune suppression downstream of IL-2 signaling (15). mTOR integrates "signal 2" of T cell activation to facilitate metabolic changes needed to support cellular growth and proliferation. Rapamycin disrupts formation of mTOR complex 1 (mTORC1), which mediates these effects (ref. 15 and 
A

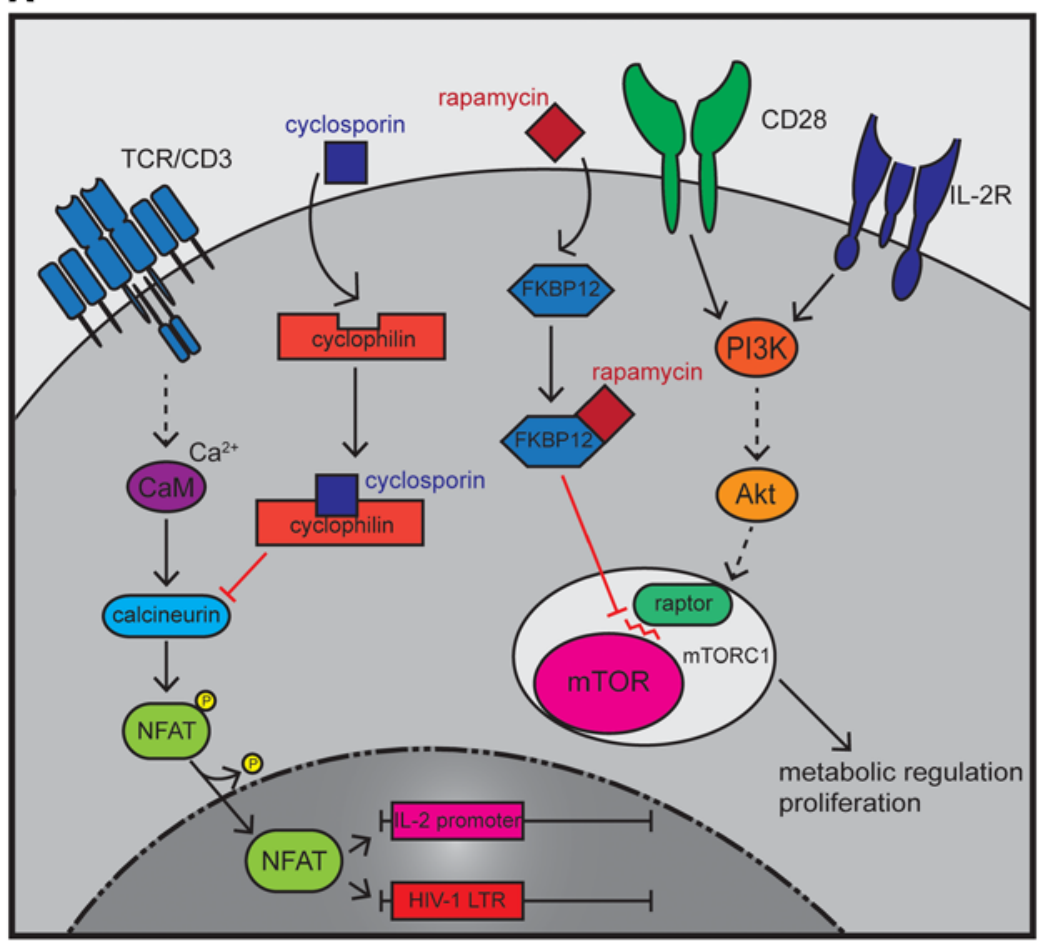

B

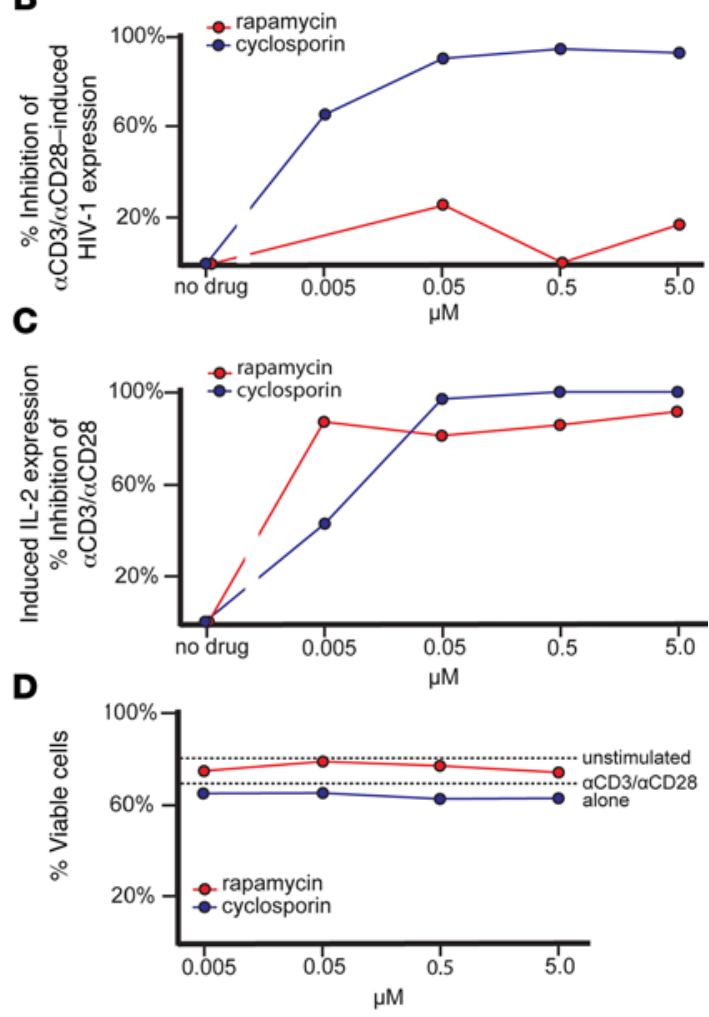

Figure 1. Immunosuppressant mechanisms and dose-response inhibition of HIV-1 or cytokine expression by rapamycin or cyclosporin treatment. (A) Accepted mechanisms of action and downstream effects of rapamycin and cyclosporin. (B) qRT-PCR measurements of intracellular HIV-1 mRNA from rCD4s derived from infected individuals, shown as percentage inhibition of $\alpha C D 3 / \alpha C D 28$-induced HIV-1 expression by rapamycin or cyclosporin cotreatment. (C) Dose-dependent inhibition of $\alpha \mathrm{CD} 3 / \alpha \mathrm{CD} 28$-induced supernatant IL-2 by increasing concentrations of rapamycin or cyclosporin. (D) Effects of rapamycin or cyclosporin viability of rCD4s stimulated with $\alpha \mathrm{CD} 3 / \alpha \mathrm{CD} 28$, compared with $\alpha \mathrm{CD} 3 / \alpha \mathrm{CD} 28$ treatment alone or unstimulated cells. Data points are the average of duplicate experiment conditions.

Figure 1A). Importantly, rapamycin does not affect mTOR complex 2 (mTORC2), which positively regulates PKC activation and downstream pathways in $\mathrm{T}$ cell activation $(15,16)$. It has been used in HIV-1-infected patients undergoing solid-organ and bone marrow transplantation (17). Rapamycin also protects mice from death after superantigen challenge (18). We hypothesized that rapamycin treatment would prevent the adverse effects of $\mathrm{T}$ cell activation without preventing upregulation of HIV-1 transcription.

Another consideration is the effect of immunosuppressive agents on CTLs. Histone deacetylase inhibitors (HDACis), an otherwise promising class of LRAs, may interfere with CTL killing (19). Cyclosporin may also inhibit CTL function owing to a dependence of CTL degranulation on calcium influx and downstream signaling (20). In contrast, rapamycin has a positive effect on the memory $\mathrm{CD}^{+} \mathrm{T}$ cell response to viral infection (21). We tested whether rapamycin interferes with CTL killing of infected cells in the context of HIV-1 shock-and-kill.

\section{Results and Discussion}

To determine whether rapamycin affects HIV-1 transcription elicited by $\mathrm{T}$ cell activation, we treated purified $\mathrm{rCD} 4 \mathrm{~s}$ from infected individuals on suppressive cART with latencyreversing stimuli and quantitated changes in HIV-1 gene expression. This system more closely approximates the in vivo response than do latency models involving transformed cell lines or primary cells infected in vitro (8). Because the in vivo frequency of latently infected cells is low, large numbers of cells must be evaluated with sensitive assays for HIV-1 RNA. We treated aliquots of $5 \times 10^{6}$ purified $\mathrm{rCD} 4 \mathrm{~s}$ with T cell-activating stimuli in the presence of varying concentrations of immunosuppressants to determine the concentrations that blocked cytokine production without cellular toxicity. After 24 hours, intracellular HIV-1 mRNA was measured by quantitative reverse transcriptase PCR (qRT-PCR) using primers that detect all correctly polyadenylated HIV-1 transcripts (8). At concentrations as low as $50 \mathrm{nM}$, cyclosporin inhibited HIV-1 induction by $\alpha \mathrm{CD} 3 / \alpha \mathrm{CD} 28$ (Figure 1B) and IL-2 release (Figure 1C). In sharp contrast, rapamycin did not produce a dose-dependent inhibition of reactivation of HIV-1 from latency even at concentrations as high as $5 \mu \mathrm{M}$, despite inhibiting IL-2 production at lower concentrations (Figure 1, B and C). Neither drug affected cell viability in the concentration range tested (Figure 1D).

Using dose-response data from the above experiments and published studies $(14,18)$, we compared the effects of cyclosporin and rapamycin in cells from patients on cART by treating $5 \times$ $10^{6}$ cells per condition with vehicle alone (DMSO), $\alpha \mathrm{CD} 3 / \alpha \mathrm{CD} 28$ alone, or $\alpha \mathrm{CD} 3 / \alpha \mathrm{CD} 28$ plus rapamycin or cyclosporin. HIV-1 mRNA levels increased significantly after treatment with $\alpha \mathrm{CD} 3$ / 
A

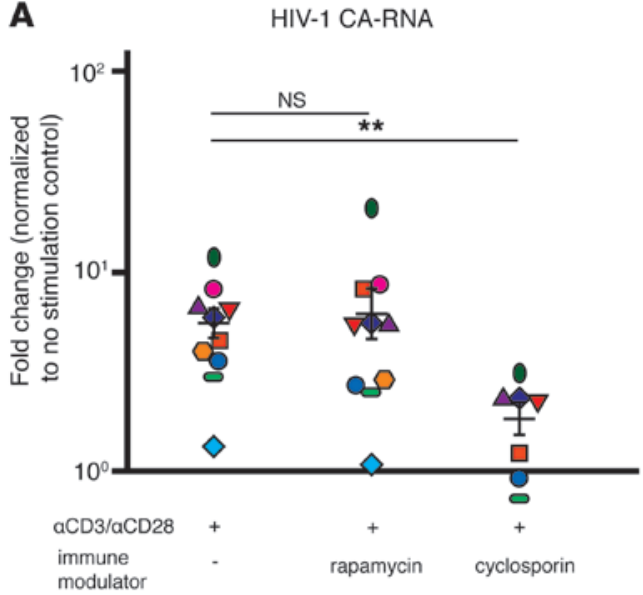

C

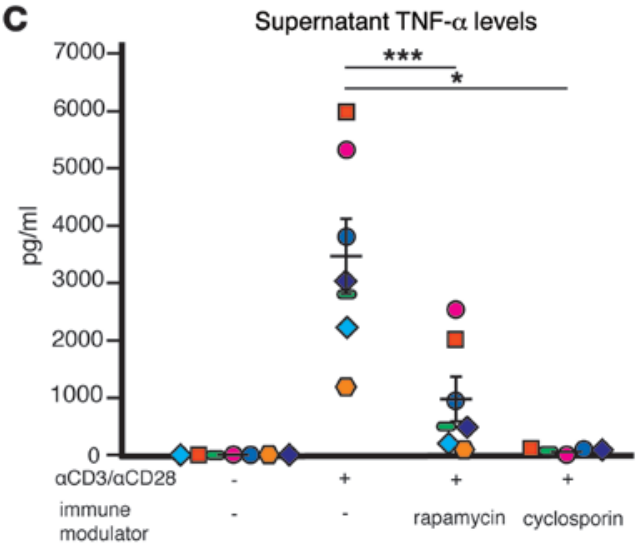

B

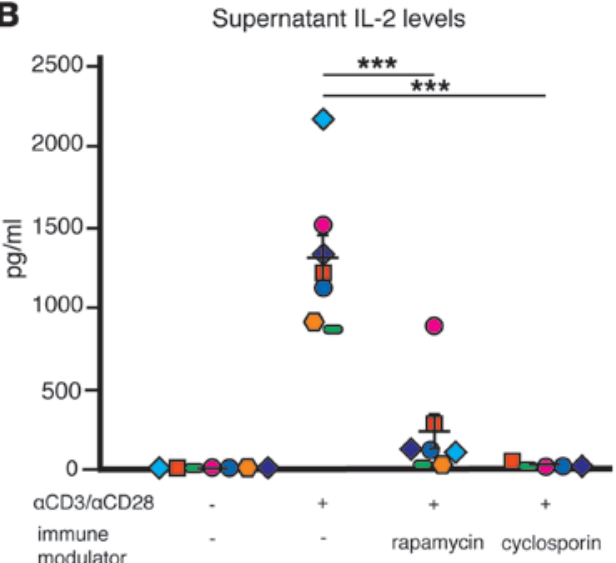

D

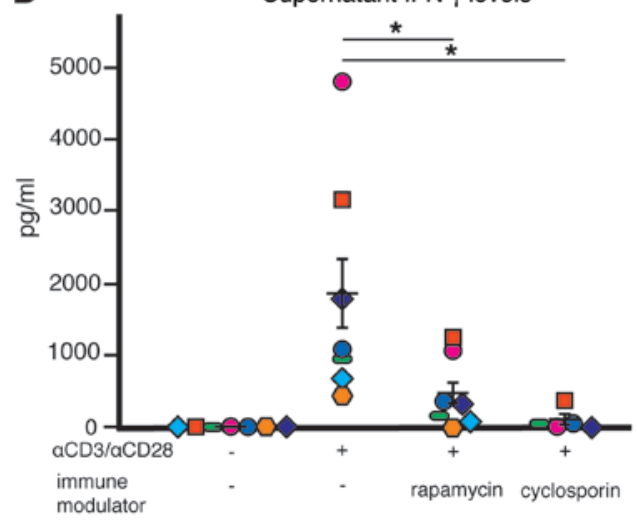

$\checkmark$ patient 1

apatient 2

o patient 3

O patient 4

O patient 5

patient 6

patient 7

- patient 8

$\Delta$ patient 9

$\nabla$ patient 10

Figure 2. Effects of immunosuppressants on $\alpha \mathrm{CD} 3 / \alpha \mathrm{CD} 28$-mediated HIV-1 expression and proinflammatory cytokine release from infected rCD4s. (A) Relative amounts of induced HIV-1 mRNA shown as fold change relative to no stimulation (DMSO alone). All samples represent qRT-PCR measurements of cell associated RNA (CA-RNA) from rCD4s derived from infected individuals $(n=10)$. (B) Effect of immunosuppressants on $\alpha$ CD3/ $\alpha$ CD28-induced rCD4 production of proinflammatory cytokine IL-2. Points represent supernatant samples from experiments described in $\mathbf{A}(n=7)$. (C) Effect of immunosuppressants on $\alpha \mathrm{CD} 3 / \alpha \mathrm{CD} 28$-induced TNF- $\alpha$ release $(n=7)$. (D) Effect of immunosuppressants on $\alpha \mathrm{CD} 3 / \alpha \mathrm{CD} 28$-induced IFN- $\gamma$ release $(n=7)$. (E) Effect of immunosuppressant cotreatment on T cell proliferation. Healthy donor PBMCs $(n=3)$ were stained with CFSE before stimulation. Data points are the average of duplicate experiment conditions. Two-tailed paired Student's $t$ test was used to determine statistical significance $\left({ }^{*} P<0.05,{ }^{* *} P<0.01,{ }^{* * *} P<0.001\right)$.

$\alpha \mathrm{CD} 28$ alone for 24 hours $(P=0.048$; Supplemental Figure $1 \mathrm{~A}$; supplemental material available online with this article; https:// doi.org/10.1172/JCI89552DS1). To account for substantial interpatient baseline variability, the data were normalized to DMSO control (Figure 2A). We observed no significant difference in HIV-1 RNA induction by $\alpha \mathrm{CD} 3 / \alpha \mathrm{CD} 28$ with or without cotreatment with $5 \mu \mathrm{M}$ rapamycin. However, cyclosporin significantly suppressed $\alpha \mathrm{CD} 3 / \alpha \mathrm{CD} 28$-induced HIV-1 mRNA induction even at a 10 -fold lower concentration $(500 \mathrm{nM})(P=0.003)$. To rule out effects of immunosuppressants on baseline HIV-1 transcription, we treated cells with vehicle alone (DMSO), rapamycin, cyclosporin, or $\alpha \mathrm{CD} 3 / \alpha \mathrm{CD} 28$ for 24 hours. $\alpha \mathrm{CD} 3 / \alpha \mathrm{CD} 28$ significantly increased HIV-1 mRNA transcripts $(P=0.008)$, while rapamycin or cyclosporin had no effect (Supplemental Figure 1B).

We also examined supernatants from the cultures described in Figure 2A for proinflammatory cytokines. IL-2, TNF- $\alpha$, and IFN- $\gamma$ were induced at high levels in $\mathrm{rCD} 4 \mathrm{~s}$, whereas other cytokines tested were not substantially produced after $\alpha \mathrm{CD} 3 / \alpha \mathrm{CD} 28$ treatment. Cotreatment with $\alpha \mathrm{CD} 3 / \alpha \mathrm{CD} 28$ and rapamycin sig- nificantly decreased release of IL-2, TNF- $\alpha$, and IFN- $\gamma$ compared with $\alpha \mathrm{CD} 3 / \alpha \mathrm{CD} 28$ alone in these $\mathrm{rCD} 4 \mathrm{~s}(P=0.0009,0.0002$, and 0.019, respectively; Figure 2, B-D). Cyclosporin treatment also significantly decreased production of these cytokines (IL-2, $P=0.001$; TNF- $\alpha, P=0.012 ;$ IFN- $\gamma, P=0.029)$. Cotreatment also inhibited production of IL-2, MCP-1, MIP- $1 \alpha$, IL-1 $\beta$, MIP- $\beta$, IFN- $\gamma$, TNF- $\alpha$, and IL- 6 by unfractionated peripheral blood mononuclear cells (PBMCs) (Supplemental Figure 2A). Rapamycin inhibited MIP- $1 \alpha$ production by over $50 \%$ and all other measured cytokines by over $80 \%$ (Supplemental Figure 2B). This effect was not due to cellular toxicity (Supplemental Figure 3A).

We then measured the effect of each treatment on cell proliferation, another potential indicator of clinical toxicity, using a CFSE assay with healthy donor PBMCs. We treated $5 \times 10^{6}$ cells per condition with DMSO alone, $\alpha \mathrm{CD} 3 / \alpha \mathrm{CD} 28$, or $\alpha \mathrm{CD} 3 / \alpha \mathrm{CD} 28$ and rapamycin or cyclosporin for 24 hours (Supplemental Figure 3B). $\alpha C D 3$ / $\alpha \mathrm{CD} 28$ stimulation caused significant proliferation $(P=0.014)$ that was substantially inhibited by rapamycin and cyclosporin $(P=0.012$ and 0.018, respectively) (Figure 2E). 
A

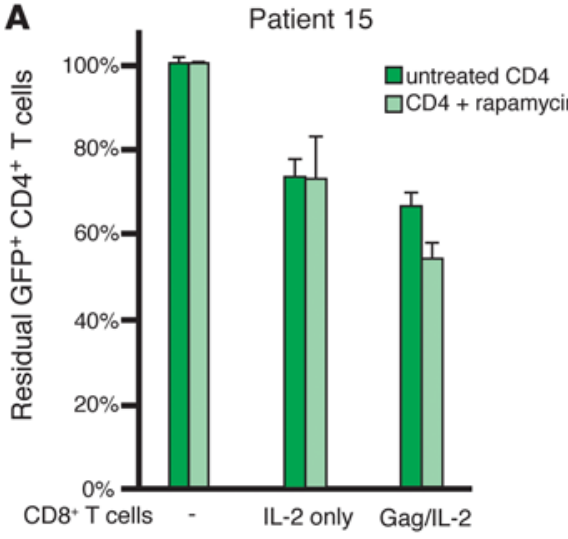

Patient 16

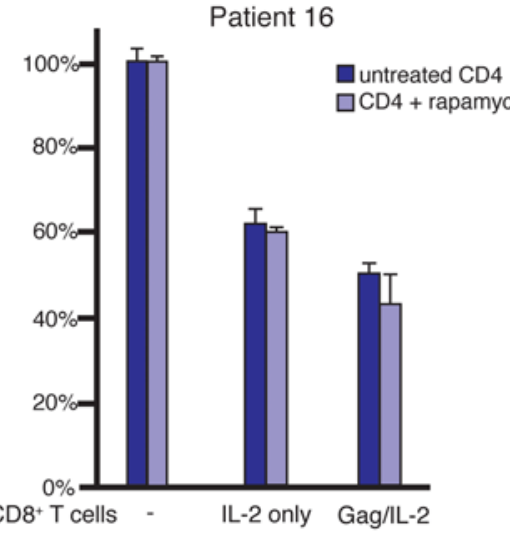

Patient 22

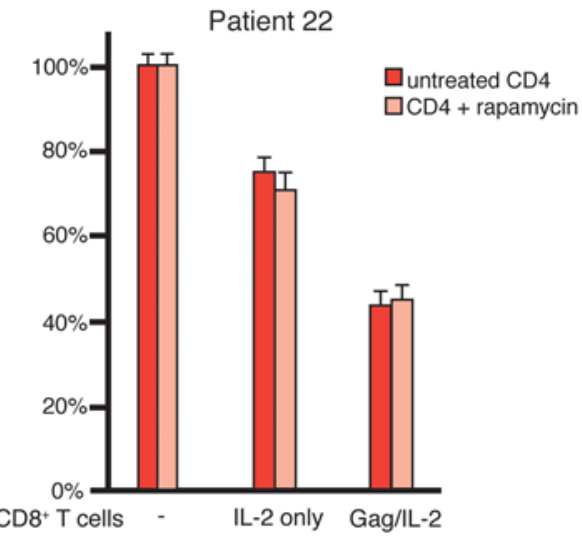

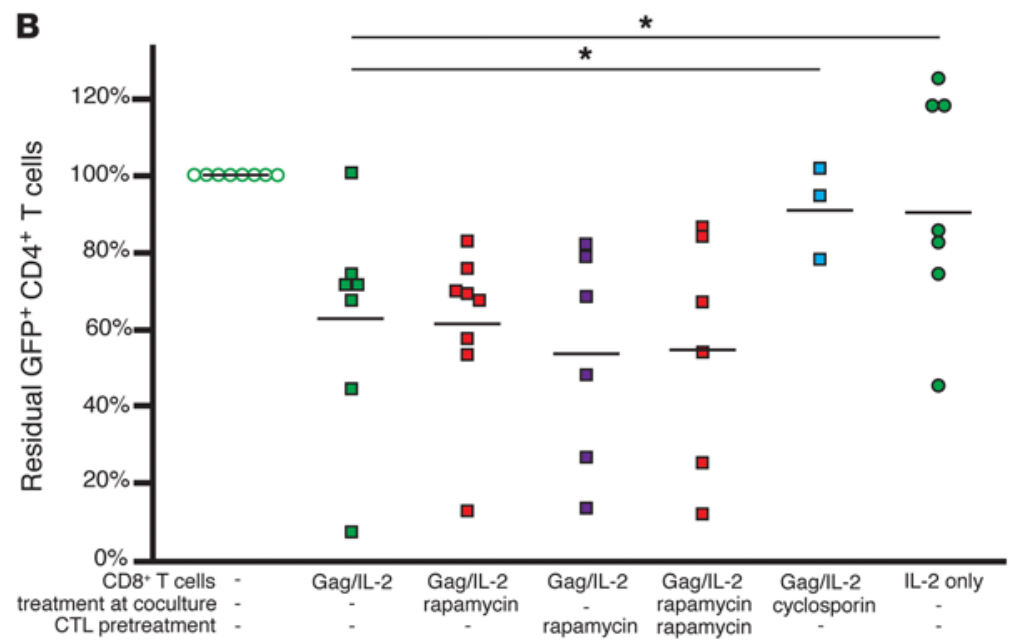

Figure 3. Rapamycin treatment does not reduce CTL killing. (A) Rapamycin does not affect viral peptide production and presentation of epitopes to CTLs $(n=3)$. Activated CD4+ T cells were infected with NL4-3- $\Delta$ env-GFP and cultured in media alone, or with rapamycin for 24 hours before coculture. Infected $\mathrm{CD} 4^{+} \mathrm{T}$ cells were cocultured at a 1:1 $\mathrm{E} / \mathrm{T}$ ratio with $\mathrm{CD} 8^{+} \mathrm{T}$ cells prestimulated with IL-2 alone or Gag and IL-2. After 72 hours, reduction in infected cells was measured by flow cytometry. (B) CTL-mediated killing of HIV-1-infected cells is impaired by the presence of cyclosporin, but not rapamycin ( $n=8$ ). As above, $C D 4^{+} T$ cells were infected with NL4-3- $\Delta$ env-GFP. Infected CD4 ${ }^{+} T$ cells were cultured alone, or cocultured at a 1:1 E/T ratio with CD8 ${ }^{+} T$ cells. As indicated, some CD8 ${ }^{+} \mathrm{T}$ cells were pretreated with rapamycin for 3 days before coculture, or cocultured in the presence of rapamycin or cyclosporin. Data points are the average of duplicate experiment conditions. Two-tailed paired Student's $t$ test was used to determine statistical significance $\left({ }^{*} P<0.05\right)$.

$\alpha \mathrm{CD} 3 / \alpha \mathrm{CD} 28$ treatment strongly induced activation marker expression on PBMCs from infected individuals after 24 hours. Cotreatment with rapamycin decreased expression of CD25 and PD-1 $(P=0.024$ and 0.016), as did cyclosporin $(\mathrm{CD} 25, P=0.009$, and PD-1, $P=0.004$ ) (Supplemental Figure $3 C$ ), suggesting that immunosuppressant treatment can downregulate chronic inflammation and $\mathrm{T}$ cell exhaustion, respectively. Together, these data demonstrate that rapamycin decouples latency reversal and cytokine production, allowing maximal HIV-1 induction while preventing toxic consequences of $\mathrm{T}$ cell activation. In contrast, cyclosporin was not suitable because of inhibition of HIV-1 gene expression.

We next tested the effect of rapamycin on a class of LRAs that may induce some level of immune activation: PKC agonists with and without HDACi $(10,11)$. Rapamycin did not affect HIV-1 mRNA induction by the PKC agonist bryostatin-1, the HDACi romidepsin, or the combination of these (Supplemental Figure 5, A and B). A modest increase in HIV-1 mRNA was seen with bryostatin-1 or romidepsin alone compared with a more substantial induction by the combination, consistent with previous reports $(8$,
22). We also tested the release of cytokines suggested to be associated with bryostatin-1 administration in vivo (23). Similar to the robust inhibition of PBMC cytokine release induced by $\alpha \mathrm{CD} 3 /$ $\alpha \mathrm{CD} 28$ treatment seen above, IL-8 and MIP-1 $\alpha$ were substantially $(>50 \%)$ reduced by addition of rapamycin, whereas IL-1 $1 \beta$, IL- 6 , and TNF- $\alpha$ were not significantly inhibited (Supplemental Figure 5C). In contrast to global immune activation by stimuli including $\alpha \mathrm{CD} 3 / \alpha \mathrm{CD} 28$, it is unclear whether cytokine release is related to in vivo toxicity of bryostatin-1. However, these results demonstrate the potential for rapamycin to have a wide applicability to different latency-reversing approaches.

To further assess the feasibility of rapamycin use in cure strategies, we tested whether it affected CTL killing of infected $\mathrm{CD} 4^{+}$ T cells using a previously described coculture system (detailed setup in Supplemental Figure 4 and ref. 24). Activated $\mathrm{CD} 4^{+} \mathrm{T}$ cells from infected donors were superinfected with an HIV-1 reporter virus expressing GFP (NL4-3- $\Delta$ env-GFP) and then cocultured at a 1:1 effector-to-target (E/T) ratio with autologous $\mathrm{CD} 8^{+} \mathrm{T}$ cells that had been prestimulated with a Gag peptide mixture and IL-2 for 6 days. 
For each patient, a decrease in $\mathrm{GFP}^{+} \mathrm{CD} 4^{+}$cells was observed when infected $\mathrm{CD}^{+} \mathrm{T}$ cells were cocultured with prestimulated $\mathrm{CD} 8^{+}$ $\mathrm{T}$ cells, compared with infected $\mathrm{CD} 4^{+} \mathrm{T}$ cells cultured alone. $\mathrm{CD} 8^{+}$ $T$ cells pretreated with IL-2 alone caused a less substantial reduction in $\mathrm{GFP}^{+}$cells. Treating the infected $\mathrm{CD}^{+} \mathrm{T}$ cells with rapamycin for 24 hours before coculture did not prevent CTL killing, showing that epitope presentation by infected $\mathrm{CD} 4^{+} \mathrm{T}$ cells was not affected (Figure 3A and Supplemental Figure 3D). We next tested whether rapamycin inhibited CTL killing of targets presenting HIV-1 epitopes using the above coculture system. Coculture with $\mathrm{CD} 8^{+} \mathrm{T}$ cells prestimulated with IL-2 plus Gag peptides caused significant elimination of $\mathrm{GFP}^{+} \mathrm{CD}^{+} \mathrm{T}$ cells, compared with $\mathrm{CD}^{+} \mathrm{T}$ cells prestimulated with IL-2 alone $(P=0.047)$. Rapamycin added at the time of coculture did not affect CTL-mediated killing of infected $\mathrm{CD} 4^{+} \mathrm{T}$ cells, whereas cyclosporin had the expected inhibitory effect $(P=0.027)$ (Figure 3B). In addition, pretreatment of $\mathrm{CD}^{+} \mathrm{T}$ cells with rapamycin for 3 days before coculture had no effect on the killing of infected cells (Figure 3B). These findings are consistent with previous reports of the effect of these immunosuppressants on CTLs $(20,21)$.

Overall, our results show that rapamycin inhibits cytokine release and cellular proliferation but does not affect HIV-1 latency reversal or CTL-mediated killing of HIV-1-infected cells. Interestingly, Stock et al. (17) reported that HIV-1-infected kidney transplant recipients who received rapamycin (sirolimus) for immunosuppression had lower levels of proviral DNA in PBMCs compared with HIV-1-infected transplant recipients who received cyclosporin or tacrolimus, both calcineurin inhibitors. We propose that the high levels of immune activation induced by transplantation (25) could reactivate expression of latent HIV-1. Our data indicate that HIV-1 transcription could occur in patients on rapamycin, allowing elimination of latently infected cells through viral cytopathic effects or cell-mediated cytotoxicity, leaving rapamycin recipients with a long-term reduction in HIV-1 DNA levels. In contrast, HIV-1 gene expression would be inhibited in individuals receiving calcineurin inhibitors including cyclosporin. This clinical finding suggests that our ex vivo results may be applicable to HIV-1-infected patients requiring solid-organ transplant.

In the search for effective approaches for reactivating latent HIV-1, T cell activation continues to be the standard for maximal transcriptional activation of latent proviruses. However, compounds that elicit $\mathrm{T}$ cell activation markers are often overlooked in favor of less effective LRAs. To avoid this compromise, we test- ed a latency reversal strategy that allows signaling downstream of TCR stimulation to effectively activate HIV-1 gene expression while controlling toxicity by cotreatment with the MTOR inhibitor rapamycin. We hypothesize that previously discarded strategies eliciting T cell activation could allow for effective reversal of HIV-1 latency in infected individuals on effective cART who are also treated with mTOR-inhibiting drugs such as rapamycin. These findings may allow an expansion in the repertoire of clinically relevant LRAs currently being investigated.

\section{Methods}

Statistics. Two-tailed paired Student's $t$ test was used to determine statistical significance where indicated. Error bars represent mean \pm SEM. We considered $P$ less than 0.05 to be statistically significant.

Study approval. The study was approved by the Johns Hopkins Institutional Review Board. Written informed consent was obtained from all participants.

A complete, detailed description of methods can be found in the supplemental material.

\section{Author contributions}

ARM and RFS conceived of the study and designed experiments. ARM and RAP conducted experiments and analyzed data. CMD and RFA obtained institutional review board approval and managed study participant recruitment. AC recruited study participants. ARM and RFS wrote the manuscript.

\section{Acknowledgments}

We thank all study participants for making this research possible. We also thank Pablo Tebas, Luis Montaner, Soe Yu Naing, and Subul Beg for assisting with study participants, and Larry Moulton and Daniel Rosenbloom for statistical advice. This work was supported by UM1AI126620 (co-funded by NIAID, NIMH, NINDS, and NIDA), the Martin Delaney CARE and DARE Collaboratories (NIH grants AI096113 and 1U19AI096109), by the Johns Hopkins Center for AIDS Research (P30AI094189), by NIH grant 43222, and by the Howard Hughes Medical Institute and the Bill and Melinda Gates Foundation.

Address correspondence to: Robert F. Siliciano, Johns Hopkins University School of Medicine, 1721 E. Madison Street, Baltimore, Maryland 21205, USA. Phone: 410.955.2958; E-mail:rsiliciano@jhmi.edu.
1. Finzi D, et al. Identification of a reservoir for HIV-1 in patients on highly active antiretroviral therapy. Science. 1997;278(5341):1295-1300.

2. Chun TW, et al. Presence of an inducible HIV-1 latent reservoir during highly active antiretroviral therapy. Proc Natl Acad Sci US A. 1997;94(24):13193-13197.

3. Siliciano JD, et al. Long-term follow-up studies confirm the stability of the latent reservoir for HIV-1 in resting CD4 ${ }^{+} \mathrm{T}$ cells. Nat Med. 2003;9(6):727-728.

4. Archin NM, et al. Administration of vorinostat disrupts HIV-1 latency in patients on antiretroviral therapy. Nature. 2012;487(7408):482-485.

5. Deeks SG. HIV: shock and kill. Nature. 2012;487(7408):439-440.
6. Prins JM, et al. Immuno-activation with anti-CD3 and recombinant human IL-2 in HIV-1-infected patients on potent antiretroviral therapy. AIDS. 1999;13(17):2405-2410.

7. Spivak AM, Planelles V. HIV-1 eradication: early trials (and tribulations). Trends Mol Med. 2016;22(1):10-27.

8. Bullen CK, Laird GM, Durand CM, Siliciano JD, Siliciano RF. New ex vivo approaches distinguish effective and ineffective single agents for reversing HIV-1 latency in vivo. Nat Med. 2014;20(4):425-429.

9. Isakov N, Altman A. Protein kinase C(theta) in T cell activation. Annu Rev Immunol. 2002;20:761-794.

10. Stach CS, Herrera A, Schlievert PM. Staphylococcal superantigens interact with multiple host receptors to cause serious diseases. Immunol Res. 2014;59(1-3):177-181.

11. Stebbings R, Eastwood D, Poole S, Thorpe R. After TGN1412: recent developments in cytokine release assays. JImmunotoxicol. 2013;10(1):75-82.

12. Williams SA, Greene WC. Regulation of HIV-1 latency by T-cell activation. Cytokine. 2007;39(1):63-74.

13. Chow CW, Rincón M, Davis RJ. Requirement for transcription factor NFAT in interleukin-2 expression. Mol Cell Biol. 1999;19(3):2300-2307.

14. Chan JK, Bhattacharyya D, Lassen KG, Ruelas D, Greene WC. Calcium/calcineurin synergizes with prostratin to promote NF- $\kappa \mathrm{B}$ dependent activation of latent HIV. PLoS One. 2013;8(10):e77749. 
15. Powell JD, Pollizzi KN, Heikamp EB, Horton MR. Regulation of immune responses by mTOR. Annu Rev Immunol. 2012;30:39-68.

16. Thomson AW, Turnquist HR, Raimondi G. Immunoregulatory functions of mTOR inhibition. Nat Rev Immunol. 2009;9(5):324-337.

17. Stock PG, et al. Reduction of HIV persistence following transplantation in HIV-infected kidney transplant recipients. Am J Transplant. 2014;14(5):1136-1141.

18. Krakauer T, Buckley M, Issaq HJ, Fox SD. Rapamycin protects mice from staphylococcal enterotoxin B-induced toxic shock and blocks cytokine release in vitro and in vivo. Antimicrob Agents Chemother. 2010;54(3):1125-1131.
19. Jones RB, et al. Histone deacetylase inhibitors impair the elimination of HIV-infected cells by cytotoxic T-lymphocytes. PLoS Pathog. 2014;10(8):e1004287.

20. Grybko MJ, Bartnik JP, Wurth GA, PoresFernando AT, Zweifach A. Calcineurin activation is only one calcium-dependent step in cytotoxic $\mathrm{T}$ lymphocyte granule exocytosis. J Biol Chem. 2007;282(25):18009-18017.

21. Araki K, et al. mTOR regulates memory CD8 T-cell differentiation. Nature. 2009;460(7251):108-112.

22. Laird GM, et al. Ex vivo analysis identifies effective HIV-1 latency-reversing drug combinations. JClin Invest. 2015;125(5):1901-1912.
23. Bosco MC, Rottschafer S, Taylor LS, Ortaldo JR, Longo DL, Espinoza-Delgado I. The antineoplastic agent bryostatin-1 induces proinflammatory cytokine production in human monocytes: synergy with interleukin-2 and modulation of interleukin-2R $\gamma$ chain expression. Blood. 1997;89(9):3402-3411.

24. Shan L, et al. Stimulation of HIV-1-specific cytolytic T lymphocytes facilitates elimination of latent viral reservoir after virus reactivation. Immunity. 2012;36(3):491-501.

25. Jiang S, Herrera O, Lechler RI. New spectrum of allorecognition pathways: implications for graft rejection and transplantation tolerance. Curr Opin Immunol. 2004;16(5):550-557. 